\title{
Modelo Empírico, Descriptivo y Predictivo Para el Compromiso de Identidad en el Chat en Adolescentes Escolares Chilenos
}

\section{Empirical, Descriptive and Predictive Model for Identity Involvement in Chat use by Chilean School Adolescents}

\author{
María Paz Altuzarra \\ Pontificia Universidad Católica de Chile \\ Beatriz Zegers \\ Universidad de los Andes
}

\begin{abstract}
Se desarrolla un modelo empírico, descriptivo y predictivo de regresión múltiple de las relaciones entre compromiso de identidad en el Chat y salud mental, sexo, curso y tipo de Chat en una muestra de 385 alumnos de ambos sexos, de I, II, III y IV Medio de tres colegios de Santiago de Chile. Se aplicó un cuestionario sobre uso de Internet, Escala de Compromiso de Identidad en el Chat y Cuestionario de Salud Mental en la Edad Juvenil. Se encontró que tanto la salud mental como el tipo de Chat predecían el compromiso de la identidad en el Chat. Se observó un mayor compromiso entre jóvenes mujeres de I y II Medio y que empleaban Chat abierto o mixto.
\end{abstract}

Palabras Clave: identidad juvenil, salud mental, chat.

An empirical descriptive and predictive model of multiple regression on the relationships between identity involvement in the Chat and mental health, sex, grade and modality of Chat in a sample of 385 male and female high school students, from Santiago de Chile, is presented. The instruments used were: a questionnaire about Internet usage, an Identity Compromise Scale in the Chat and a Youth Mental Health Scale. Results indicate that both, mental health and type of Chat, predicted compromise of identity in the Chat. It was observed a greater compromise among female teenagers from 9th and 10th grade who used open and mixed Chat.

Keywords: youth identity, mental health, chat.

\section{Identidad Juvenil, Salud Mental y Chat}

Los adolescentes del siglo XXI forman parte de la sociedad post-industrial. Ésta se encuentra regulada por relaciones que sistematizan el consumo, las organizaciones, la información, la educación y las costumbres. Los medios de comunicación posibilitan que cada cual se vuelque sobre sí mismo en busca de su verdad y bienestar (Lipovetsky, 1986). Disminuyen las certezas, los valores se relativizan, los lazos sociales se hacen laxos y no hay guías adecuadas para la orientación individual y colectiva (Sandoval, 2002). Hasta hace un siglo, las relaciones se circunscribían a una comunidad; hoy, en cambio, las tecnologías comunicacionales permiten ampliar

María Paz Altuzarra, Programa de Magíster en Psicología, Escuela de Psicología, Pontificia Universidad Católica de Chile. Beatriz Zegers, Escuela de Psicología, Universidad de los Andes, Chile.

La correspondencia relativa a este artículo deberá ser dirigida a los autores, Universidad de los Andes, San Carlos de Apoquindo 2200, Santiago de Chile. E-mail: mpaltuzarra@uandes. cl; bzegers@uandes.cl el círculo, afectando las pautas de la vida social y plantean dilemas a la identidad personal (Gergen, 1991). La tarea de la adolescencia es definir la identidad personal, por lo que resulta interesante estudiar las relaciones entre esta temática y las posibilidades que ofrece Internet de experimentar con ella a través de los Chat.

\section{Adolescencia y Definición de la Identidad}

Kroger (2000) clasifica los estudios de identidad en cuatro modelos: (a) estructurales: la conciben como una estructura interna que se desarrolla siguiendo un patrón secuencial y predecible; (b) modelos socioculturales: la vinculan a las definiciones de roles y estudian el papel que juega la sociedad en proveer o no alternativas de identidad individual (Shotter \& Gergen, 1989 citado en Kroger, 2000); (c) modelos narrativos: establecen que la identidad se traza a través de la historia de vida que cada cual escribe (McAdams, 1993); (d) modelos psicosociales: señalan que se forma en la interacción entre estructuras internas personales y demandas de un grupo de referencia social 
(Kroger, 2000). El punto de partida de estos últimos se encuentra en los trabajos de Erikson $(1959,1968)$, para quien la identidad comprende tres dimensiones: (a) psicológica o identidad del yo, (b) personal o repertorio de conductas y rasgos del carácter y (c) social o reconocimiento de roles.

La tarea de definir la identidad requiere un período de exploración, análisis y evaluación del sí mismo real e ideal (Allison \& Shultz, 2001). En éste se diferencian tres etapas: prepubertad, pubertad y adolescencia. A cada una le corresponden edades y temáticas de referencia desde la perspectiva de la identidad. En la prepubertad las preocupaciones se refieren al sí mismo corporal; en la pubertad éstas giran en torno a las preguntas “¿quién soy yo?”, “¿cuáles son mis rasgos individuales?”. Definida en parte la cara interna de la identidad, los adolescentes se preocuparán por encontrar un lugar para sí mismos en la sociedad, ejercitando roles y orientándose hacia definiciones específicas (Newman \& Newman, 1999). Definir la identidad implica desarrollar la noción del tipo de persona que se es en distintos ambientes y considerarse el mismo a lo largo del tiempo y en independencia de los lugares en que se encuentre (Côté \& Levine, 1987 citado en Van Hoof $\&$ Raaijmakers, 2002); la sociedad permite estas indagaciones al ofrecer al joven un período de prueba (moratoria) y al definir el rol de las instituciones en las cuales este proceso se desarrolla.

Erikson se refirió al fracaso que puede ocurrir en la adolescencia en la integración de las identificaciones tempranas en una identidad armónica con el término difusión de identidad; en 1959 y 1968 describió el fenómeno clínico asociado, destacando la alteración en la capacidad de intimidad y mutualidad, difusión en la perspectiva temporal, disminución en la habilidad para trabajar, hostilidad hacia los roles ofrecidos por la propia familia y conflictos con los orígenes étnicos. Siguiendo a Erikson, Akhtar (1992; Akhtar \& Samuel, 1996) se ha referido a los signos clínicos y síntomas en el área de la identidad y la auto-experiencia de la difusión de la identidad.

\section{Adolescencia y Salud Mental}

Stanley Hall (1916 citado en Muuss, 1996) caracterizó la adolescencia como un período de tensiones y conflictos derivados del surgimiento de intensas y variadas emociones. Una perspectiva análoga fue adoptada por el psicoanálisis. Específicamente, Anna Freud (1936/1985) estudió las luchas que libra el yo para dominar los conflictos instintivos; planteó que en los casos normales esas disputas llevaban a la formación del carácter y en los patológicos, a la formación de síntomas neuróticos. De estos planteamientos se extendió la visión de la adolescencia como un tiempo en el que la presencia de síntomas sería inevitable, dificultándose la distinción entre la normalidad y la patología.

Los antecedentes de la investigación empírica permiten diferenciar entre una adolescencia normal y una patológica, como han documentado Offer y Offer (1975 citado en Offer, 1991). Florenzano (1998) establece que las diferencias se basan en la duración, frecuencia e intensidad del síntoma, junto al grado de ajuste o capacidad adaptativa del joven frente a los cambios y exigencias propias de esta etapa. Almonte y Montt (1999) han señalado criterios similares.

\section{Internet Como Medio de Comunicación}

Internet ha sido construida con una arquitectura tecnológicamente abierta, lo que permite el libre acceso y limita las restricciones (Castells, 1997). La comunicación en Internet se diferencia de la que se produce cara a cara por las posibilidades de anonimato que proporciona, permite la interacción con personas de distintas latitudes, se realiza sin la mediación corporal y es relativamente inmaterial desde la perspectiva temporal (McKenna \& Bargh, 2000).

La posibilidad de cambiar nombres y emplear seudónimos, con la dificultad de ser identificado, constituiría una de las expresiones de este anonimato (Douglas \& McGarty, 2001). La teoría de la desindividuación (Diener, 1980, Zimbardo, 1969; citados en McKenna \& Bargh, 2000) permite explicar la pérdida del sentido de identidad individual, la desinhibición y la reducción del control del propio comportamiento que puede darse en Internet (Siegel, Dubrovsky, Kiesler \& McGuire, 1986; Sproull \& Kiesler, 1991 citados en Douglas \& McGarty, 2001).

La ausencia de mediación corporal puede modificar el curso de las interacciones dada la falta de claves que determinan la atracción interpersonal: expresión facial, postura y tono de voz (McKenna \& Bargh, 2000). La introducción de íconos (emoticons), diseñados para reemplazar las claves no verbales (Utz, 2000), generan cierta confusión en su interpretación y no sustituyen los rasgos expresivos de las relaciones cara a cara (Riva, 2000). 
La relativa inmaterialidad temporal reduce las posibilidades de cooperación y retroalimentación de la comunicación directa (McKenna \& Bargh, 2000). El medio virtual impone sus reglas, los discursos son preferentemente cortos, no pueden sobreponerse y su orden no tiene que ser secuencial, lo que puede conducir a intercambios argumentativos y/o hostiles (Riva, 2002).

Los jóvenes interactúan en Internet cada vez con más frecuencia (Villani 2001; Wolak, Mitchel \& Finkelhor, 2002). En Chile, el $46.2 \%$ de los escolares entre 10 y 17 años tiene computador en su casa y el $20.5 \%$ está conectado a Internet. La digitalización llega al 39\% en los colegios públicos, al $45.5 \%$ en los particulares subvencionados y al $60.6 \%$ en los establecimientos privados pagados. El $52.1 \%$ de los jóvenes que se conectan a la red se comunican vía Chat (Fundación Chile, Consultora Adimark \& Empresa de Telecomunicaciones VTR, 2004).

Los Chat se han clasificado en (a) abiertos: la comunicación se puede realizar con desconocidos; (b) cerrados: la interacción se establece con personas que aparecen en una lista confeccionada por el usuario y (c) mixtos: usan la modalidad de Chat abierto y cerrado (Sassenberg, 2002). En un Chat abierto se puede mantener el anonimato experimentando con la identidad; en un Chat cerrado es menos probable que suceda, ya que en éste se es fácilmente reconocido (Sassenberg, 2002).

Internet serviría a cuatro propósitos en la adolescencia: (a) explorar la identidad, (b) buscar relaciones de intimidad y sentido de pertenencia, (c) sondear la separación de los padres y de la familia y (d) expresar frustraciones (Suler, 1998). Maczewski (2002) agrega la experimentación con la libertad. Por ello se ha afirmado que la red permite hacer frente a estresores del desarrollo (Eppright, Allwood, Stern \& Theiss, 1999; Garrison \& Long, 1995; Kandell, 1998; Young, 1996 citados en Hall, Alex \& Parsons, 2001).

En relación al género, Morgan y Cotten (2003) afirmaron que las mujeres utilizaban más el Chat para mantener relaciones con parientes y para crear lazos con personas distantes, que los hombres. Zegers, Larraín y Trapp (2004) encontraron que universitarios hombres eran más desinhibidos, más permisivos frente a trasgresiones que vulneran la confianza interpersonal y revelaron mayor tolerancia al enmascaramiento de aspectos nucleares de la identidad, como el cambio de sexo, que las mujeres.

\section{Laboratorio Social de Experimentación de la Identidad}

Internet es un laboratorio virtual para explorar y experimentar con diferentes versiones del sí mismo sin la desaprobación y sanción que estas experiencias podrían tener en la vida social real (Allison \& Schultz, 2001; Bargh, McKenna \& Grainne, 2002; Turkle, 1995; Wallace, 1999).

Dado el anonimato, cualquiera puede describirse con rasgos físicos diferentes, simular que pertenece a otro género, sostener creencias y valores distintos a los que sustenta en la realidad. Los adolescentes pueden jugar a ser quienes ellos desean, pueden actuar utilizando disfraces para parecer atractivos (Acosta, Ciunne \& Diodatti, 2001). La identidad puede ser negociada considerando quién es uno, quién se desea ser y lo que la ciber_comunidad propone o espera de cada cual (Talamo \& Liborio, 2001). Internet se ha conceptualizado como un nuevo ambiente social, no afecta desde fuera, sino es un espacio co-construido a través de procesos de interacción social (Subrahmanyam, Smahel \& Greenfield, 2006).

Los grupos en Internet no tienen concepciones a priori o expectativas acerca del tipo de identidad o roles a los que cada cual adhiere, favoreciendo el aumento de redes sociales y el desarrollo de destrezas y habilidades sociales (McKenna \& Bargh, 2000). Sin embargo, jugar con sí mismos alternativos en Internet puede afectar a las definiciones personales en sus aspectos profundos e íntimos como en los periféricos (Reid, 1998; Turkle, 1995; Wallace, 1999).

Zegers y Larraín (2002) han analizado, siguiendo a Akhtar (1992; Akhtar \& Samuel, 1996), ciertas dimensiones de la identidad que podrían afectarse por las experiencias en Internet. Han mostrado cómo la posibilidad de crear personajes puede estimular sentimientos de confusión; cómo la simulación, el engaño y ser engañado puede afectar la genuinidad y autenticidad; cómo el cambio de género puede despertar vivencias de confusión respecto de la claridad subjetiva en relación al propio sexo y cómo la desindividuación puede comprometer la solidaridad interna con los ideales morales característica de la identidad integrada.

\section{Internet y Salud Mental}

Los adolescentes integrados socialmente usan el Chat fortaleciendo sus contactos o buscando otros nuevos; pero, cuando existen sentimientos crónicos o transitorios de malestar o desapego social, puede usarse para evitar la soledad (Gross, Juvonen \& Ga- 
ble, 2002); para escapar a las tareas que el desarrollo plantea, lo que a su vez, limita la preparación para enfrentar las vicisitudes que conllevan las relaciones interpersonales en el mundo real. Se pueden sustituir los contactos cara a cara por experiencias sociales en línea y/o suplantar la identidad personal con pseudo identidades en línea (Young, 1998).

El uso del Chat tiene efectos beneficiosos en el bienestar de estudiantes universitarios al disminuir los síntomas depresivos, el sentimiento de soledad y al aumentar el apoyo social percibido (Morgan \& Cotten, 2003; Shaw \& Grant, 2002), visión que es compartida por Kiesler (citado en Young \& Rodgers, 1998).

En contraposición, Viñas, Villar, Caparrós, Juan, Pérez y Cornellá (2002), concluyeron que el uso del Chat se asociaría a presencia de sintomatología depresiva, alteraciones del sueño y pérdida de apetito, insatisfacción en las relaciones familiares, de pareja y, en la capacidad o habilidad para mantener relaciones sociales. Kart (1998 citado en Sanders, Field, Diego \& Kaplan, 2000) encontró, al cabo de dos años de haber entregado un computador y proporcionar instrucciones sobre el uso de Internet a familias seleccionadas al azar, una disminución de la comunicación familiar y una reducción en el círculo social cercano; los sentimientos de soledad y depresión aumentaron.

$\mathrm{Al}$ desaparecer las claves sociales que revelan ansiedad, Internet sería un lugar más seguro, confiable y menos amenazante que la vida real para personas que presentan ansiedad social (McKenna \& Bargh, 2000). Scealy, Phillips y Stevenson (2002) señalan que otros pueden buscar compensaciones a la falta de relaciones en el mundo real, favoreciendo el aislamiento social y llevar a un uso patológico de Internet.

Aunque no estudiamos las posibles relaciones entre salud mental y uso patológico de Internet, estimamos relevante referirnos a lo comunicado por Estallo (2001), quien afirma que no se debe minimizar la posibilidad de que exista un trastorno o situación disfuncional previa al abuso de la red, siendo necesario diferenciar si la adicción se debe a sus trastornos de base o si por la adicción a la red se desarrollan dichas alteraciones.

Las investigaciones precedentes no incluyen la identidad. Como su definición constituye un aspecto central del desarrollo adolescente e Internet ofrece posibilidades de experimentar con ella, es que decidimos abordar su estudio. Esta perspectiva, nos parece, puede aportar a comprender el impacto de Internet en la definición de la identidad juvenil; además, puede cooperar al esclarecimiento de la problemática aludida por Estallo (2001) y contribuir a identificar empíricamente las diferencias individuales que median y moderan las fuerzas situacionales de Internet (McKenna \& Bargh, 2000).

El objetivo general de la investigación fue desarrollar un modelo empírico, descriptivo y predictivo de las relaciones entre compromiso de la identidad en el Chat y posibles variables predictoras. Se buscó: (a) establecer si los jóvenes comprometían o no la identidad según salud mental, sexo, curso, tipo de Chat y horas de chateo; (b) comparar el grado de compromiso de la identidad en el Chat según probabilidad de presentar psicopatología, sexo, edad y tipo de Chat; y (c) establecer si existía alguna asociación entre la probabilidad de presentar síntomas psicopatológicos, según sexo, curso y tipo de Chat.

\section{Método}

\section{Participantes}

La población estuvo constituida por 433 alumnos de I, II, III y IV Medio procedentes del Liceo Amanda Labarca y Colegio Antártica Chilena de la Municipalidad de Vitacura y Colegio San Francisco del Alba de la Municipalidad de Las Condes de Santiago de Chile. Los criterios de inclusión fueron la edad y uso del Chat. Se formó una muestra intencionada de 385 alumnos de I, II, III y IV Medio, de entre 14 y 18 años; 200 mujeres y 185 hombres que utilizaban Chat, ya que 48 señalaron no chatear (Tabla 1).

\section{Procedimiento}

La Dirección de Servicios de Salud y Educación de la Municipalidad de Vitacura y de Las Condes de Santiago de Chile, designaron los colegios del estudio. Los participantes firmaron un consentimiento informado que contenía los objetivos de la investigación y establecía la confidencialidad de los datos. Los instrumentos se aplicaron en las salas de clases, en forma colectiva. La recolección de datos duró aproximadamente 60 minutos en cada curso.

\section{Instrumentos}

A los jóvenes de este estudio se les aplicaron los siguientes instrumentos:

Cuestionario sobre el uso de Internet: Consigna las variables tipo de Chat empleado (abierto, mixto y/o cerrado), horas de uso (diarias y semanales), edad, curso y el sexo (Altuzarra, 2006).

Escala de Compromiso de la Identidad en el Chat (ECICH) (Zegers, Larraín, Trapp, Avilés, Grez \& De St. Aubin, 2006). Evalúa la variable compromiso de Identidad en el Chat, a través de preguntas tales como: "Yo he engañado a otros en el Chat respecto de quién soy", "Me gusta jugar en el Chat porque puedo ser una persona distinta a la que soy en la vida real". Consta de 45 afirmaciones y se contestan en una escala de cinco puntos (nunca es cierto, muy pocas veces es cierto, algunas veces es cierto, casi 
siempre es cierto y siempre es cierto). Tiene una estructura de tres factores (Consistencia del comportamiento entre la vida real y realidad virtual, Inautenticidad y Engaño, Experimentación y Desinhibición) los que en su conjunto explican un $36.77 \%$ de varianza total del instrumento. El coeficiente alpha de Cronbach para la escala total es de 0.93 ; para los tres factores son: factor 1 : 0.82 , factor 2: 0.83 y factor $3: 0.79$. Como no cuenta con un punto de corte para diferenciar entre quienes comprometen su identidad en el Chat y aquellos que no lo hacen, en esta investigación éste se fijó en 86 puntos, valor que correspondía a la media de puntajes más una desviación estándar encontrada en la investigación de Zegers et al. (2006). Es decir, los puntajes mayores a 87 se consideraron que apuntaban a un mayor compromiso de identidad, mientras que los menores, se orientaban a uno menor. Esta categorización se utilizó cuando se establecieron relaciones entre el compromiso de identidad en el Chat y variables categóricas. En los otros casos (cálculo de regresión, ANOVAy $t$ de Student), se consideraron los puntajes totales.

Cuestionario de Salud Mental en la Edad Juvenil (CSMEJ) (Zegers, Manzi, Florenzano, Acle, Berger, González et al., 1987). Realiza un screening de salud mental y diferencia entre normalidad y probabilidad de presentar psicopatología $(p=000)$, a través de preguntas tales como: "Últimamente ¿has podido concentrarte en lo que haces?", "En los últimos seis meses ¿te has negado a obedecer y/o acatar las reglas del colegio y/o casa?". Consta de 61 ítemes y se responden en una escala de cuatro puntos (nunca, a veces, con frecuencia, casi siempre). El coeficiente de confiabilidad por bipartición es de .94 y para la multipartición el coeficiente alpha de Cronbach es de .94. Tiene una estructura de 8 factores que en su conjunto dan cuenta del $46 \%$ de la varianza total del test, aunque las categorías diagnósticas no se distribuyen en factores independientes. El punto de corte está fijado en 19 puntos; es decir, los jóvenes que obtienen 19 puntos son considerados normales mientras que los que obtienen 20 puntos o más tendrían una alta probabilidad de presentar patología. Cuando se calculó la regresión se tomó el puntaje total del test.

\section{Resultados}

Los datos fueron analizados a través del programa SPSS versión 11.5 y se realizaron análisis descriptivos de frecuencia.

\section{Estadísticos Descriptivos}

Como se observa en la Tabla 1, del total de la muestra, un $51.9 \%$ es mujer y un $48.1 \%$, hombre. Hay una mayor tendencia a utilizar Chat cerrado $(75.8 \%)$, siendo el abierto el menos usado (2.3\%). El 52.5\% usa el Chat entre 1 y 5 horas (media de 2.19 horas, $D S 1.63)$, valor que es similar para todos los cursos $y$ en independencia de la variable sexo. Se encontró una proporción similar de uso del Chat cerrado entre

Tabla 1

Distribución de la muestra según sexo, curso, edad, tipo de Chat, horas de chateo, días semanales de uso de Chat presencia o ausencia de patología en salud mental, presencia o ausencia de compromiso de identidad en el Chat

\begin{tabular}{|c|c|c|c|c|c|c|}
\hline Sexo & $\begin{array}{l}\text { Mujer } \\
200 \\
51.9 \%\end{array}$ & $\begin{array}{l}\text { Hombre } \\
185 \\
48.1 \%\end{array}$ & & & & \\
\hline Curso & $\begin{array}{l}\text { I medio } \\
114 \\
29.6 \%\end{array}$ & $\begin{array}{l}\text { II medio } \\
116 \\
30.1 \%\end{array}$ & $\begin{array}{l}\text { III medio } \\
86 \\
22.3 \%\end{array}$ & $\begin{array}{l}\text { IV medio } \\
69 \\
17.9 \%\end{array}$ & & \\
\hline Edad & $\begin{array}{l}14 \text { años } \\
60 \\
15.6 \%\end{array}$ & $\begin{array}{l}15 \text { años } \\
119 \\
30.9 \%\end{array}$ & $\begin{array}{l}16 \text { años } \\
108 \\
28.1 \%\end{array}$ & $\begin{array}{l}17 \text { años } \\
80 \\
20.8 \%\end{array}$ & $\begin{array}{l}18 \text { años } \\
18 \\
4.7 \%\end{array}$ & \\
\hline Tipo de Chat & $\begin{array}{l}\text { Cerrado } \\
292 \\
75.8 \%\end{array}$ & $\begin{array}{l}\text { Abierto } \\
9 \\
2.3 \%\end{array}$ & $\begin{array}{l}\text { Mixto } \\
84 \\
21.8 \%\end{array}$ & & & \\
\hline $\begin{array}{l}\text { Horas } \\
\text { semanales }\end{array}$ & $\begin{array}{l}1-5 \\
202 \\
52.5 \%\end{array}$ & $\begin{array}{l}6-10 \\
69 \\
17.9 \%\end{array}$ & $\begin{array}{l}11-15 \\
38 \\
9.9 \%\end{array}$ & $\begin{array}{l}16-20 \\
19 \\
4.9 \%\end{array}$ & $\begin{array}{l}21-25 \\
28 \\
7.3 \%\end{array}$ & $\begin{array}{l}26 \text { y más } \\
29 \\
7.5 \%\end{array}$ \\
\hline $\begin{array}{l}\text { Días } \\
\text { semanales }\end{array}$ & $\begin{array}{l}1-2 \\
126 \\
82 \%\end{array}$ & $\begin{array}{l}3-4 \\
82 \\
21.3 \%\end{array}$ & $\begin{array}{l}5-6 \\
70 \\
18.2 \%\end{array}$ & $\begin{array}{l}7 \\
107 \\
27.8 \%\end{array}$ & & \\
\hline $\begin{array}{l}\text { Patología } \\
\text { Salud mental }\end{array}$ & $\begin{array}{l}\text { No presenta } \\
259 \\
67.3\end{array}$ & $\begin{array}{l}\text { Presenta } \\
126 \\
32.7 \%\end{array}$ & & & & \\
\hline $\begin{array}{l}\text { Compromiso } \\
\text { identidad }\end{array}$ & $\begin{array}{l}\text { No presenta } \\
288 \\
74.8 \%\end{array}$ & $\begin{array}{l}\text { Presenta } \\
97 \\
25.2 \%\end{array}$ & & & & \\
\hline
\end{tabular}


Tabla 2

Correlación entre puntaje escala ECICH y curso $(N=385)$

\begin{tabular}{lc}
\hline & $\begin{array}{c}\text { Correlación de } \\
\text { Pearson }\end{array}$ \\
\hline Puntaje total ECICH & $-0.025^{* *}$ \\
$\begin{array}{l}\text { Factor consistencia del } \\
\text { comportamiento }\end{array}$ & $-0.247^{* *}$ \\
$\begin{array}{l}\text { Factor inautenticidad y engaño } \\
\text { Factor experimentación y } \\
\text { deshinibición }\end{array}$ & $-0.147^{* *}$ \\
\hline
\end{tabular}

$* * p<0.01$

hombres (51\%) y mujeres (49\%). El Chat abierto es más usado por mujeres ( $77.8 \%$ vs. $22.2 \%)$ y el mixto es preferido por las mujeres (59.5\% vs. $40.5 \%)$.

\section{Compromiso de la Identidad en el Chat Según}

Sexo, Edad, Tipo de Chat y Horas de Chateo

Tal como muestra la Tabla 2, el cálculo de los coeficientes de correlación de Pearson entre la edad y los tres factores identificados en el ECICH son significativos. Para el puntaje total, la correlación de $-0.0225(p<0.001)$ indica que a mayor edad habría un menor compromiso de identidad en el Chat; para el factor Consistencia del comportamiento entre la vida real y la virtual, la correlación de -0.247 ( $p<$ 0.001 ) significa que a mayor edad la inconsistencia del comportamiento sería menor; para el factor
Inautenticidad y Engaño, la correlación de -0.147 $(p<0.001)$ señala que la autenticidad aumenta con la edad; lo mismo sucede con el factor Experimentación y Desinhibición, el cual, al correlacionarse con el curso, obtiene una correlación de $-0.184(p$ $<0.001)$. Se desprende que a mayor edad habría menor experimentación y desinhibición.

La prueba chi cuadrado de Pearson no arrojó relaciones entre compromiso de identidad en el Chat (puntaje ECICH) y sexo $\chi^{2}(1, \mathrm{~N}=385)=1.064, p$ $=0.392$; ni tampoco para horas de chateo $\chi^{2}(3, \mathrm{~N}=$ $385)=2.207, p=0.820$. Por otra parte se encontró que el tipo de Chat se relaciona con el ECICH, $\chi^{2}$ $(1, \mathrm{~N}=385)=10.068, p=0.002$.

Comparación Entre el Grado de Compromiso de la Identidad en el Chat y Curso, Tipo de Chat, Sexo y Probabilidad de Presentar Psicopatología

Al comparar el compromiso de identidad en el Chat por curso (I, II, III y IV Medio), a través del ANOVA, encontramos diferencias entre los grupos $(p=0.02)$. Las comparaciones post-hoc (Tukey) establecen que las diferencias se producen entre I y IV Medio $(p=0.009)$ y entre II y IV medio $(p=$ 0.013 ). Dado que los mayores puntajes se registran en I y II Medio se desprende que son estos alumnos los que más comprometen su identidad cuando chatean. No se observan diferencias entre el I y el II Medio y el II y el III.

Tabla 3

Análisis de la varianza para los puntajes totales del ECICH según tipo de Chat y curso

\begin{tabular}{|c|c|c|c|}
\hline Fuente & Variable dependiente & $F$ & Significación \\
\hline Tipo de Chat & Compromiso de identidad en el Chat (Puntaje total ECICH) & 7.237 & $.001 * *$ \\
\hline Curso & Compromiso de identidad en el Chat (Puntaje total ECICH) & 5.171 & $.002 * *$ \\
\hline
\end{tabular}

Tabla 4

Análisis T de Student para los puntajes totales del ECICH según puntaje CSMEJ y sexo

\begin{tabular}{llcc}
\hline \multicolumn{1}{c}{ Fuente } & \multicolumn{1}{c}{ Variable dependiente } & $T$ & Significación \\
\hline $\begin{array}{l}\text { Salud mental (puntaje CSMEJ: presencia } \\
\text { o ausencia de probabilidad de síntomas } \\
\text { psicopatológicos) }\end{array}$ & $\begin{array}{l}\text { Compromiso de identidad en el Chat (Puntaje } \\
\text { total ECICH) }\end{array}$ & 3.524 & $.000^{* *}$ \\
Sexo & $\begin{array}{l}\text { Compromiso de identidad en el Chat (Puntaje } \\
\text { total ECICH) }\end{array}$ & -1.029 & .304 \\
& & & \\
\hline
\end{tabular}

** $p<0.01$ 
Tabla 5

Regresión múltiple, puntajes de la escala ECICH, puntajes del CSMEJ, tipo de Chat, edad y sexo

\begin{tabular}{|c|c|c|c|c|c|}
\hline & \multicolumn{2}{|c|}{ Coeficientes no estandarizados } & \multirow{2}{*}{$\begin{array}{l}\text { Coeficientes estandarizados } \\
\text { Beta }\end{array}$} & \multirow[t]{2}{*}{$t$} & \multirow[t]{2}{*}{ Significación } \\
\hline & B & Error típico & & & \\
\hline (Constante) & 117.149 & 13.299 & & 8.809 & $.000^{* *}$ \\
\hline $\begin{array}{l}\text { Tipo de Chat: } \\
\text { Cerrado v/s mixto }\end{array}$ & 14.404 & 6.015 & .115 & 2.395 & $.017^{*}$ \\
\hline $\begin{array}{l}\text { Tipo de Chat: } \\
\text { Cerrado v/s abierto }\end{array}$ & 5.288 & 2.230 & .115 & 2.371 & $.018^{*}$ \\
\hline Sexo & 7.509 & 1.968 & .198 & 3.815 & $.000 * *$ \\
\hline Salud Mental & .499 & .091 & .284 & 5.493 & $.000 * *$ \\
\hline Edad del Participante & -3.417 & .831 & -.200 & -4.113 & $.000 * *$ \\
\hline
\end{tabular}

El análisis ANOVA entre compromiso de identidad en el Chat y el tipo de Chat (abierto, cerrado o mixto) mostró diferencias significativas entre los grupos $(p=0.001)$. Las comparaciones post-hoc (Tuckey) indican que existen diferencias tanto entre el Chat cerrado vs. abierto $(p=0.017)$ como entre el Chat cerrado vs. mixto $(p=0.013)$. Los que utilizan Chat abierto presentan mayor compromiso de la identidad en el Chat comparados con los que emplean Chat cerrado; similar situación ocurre con los que utilizan el Chat abierto. Los puntajes del ECICH no mostraron diferencias significativas entre aquellos que emplean Chat abierto comparados con los que interactúan a través del Chat mixto $(p=$ 0.224). De lo anterior se desprende que es en el Chat abierto y mixto donde se da el mayor compromiso de la identidad en el Chat.

El análisis de la prueba $t$ de Student para compromiso de identidad en el Chat (puntaje ECICH) con sexo, no arrojó diferencias significativas $(t=-1.029$, $p=0.304)$, pero sí encontramos diferencias significativas $(t=3.524, p=0.00)$ cuando se compara éste compromiso con salud mental (puntaje CSMEJ). Es decir, los que tienen probabilidad de presentar síntomas psicopatológicos comprometen más su identidad que quienes no presentan dicha probabilidad (IC 95\%, 3.162 - 11.143).

\section{Modelo de Regresión Entre Compromiso de la Identidad en el Chat y Salud Mental}

Dado que se encontró que las variables curso, tipo de Chat, sexo y salud mental (puntaje CSMEJ) eran posibles predictores de la variable criterio: compromiso de la identidad en el Chat (puntaje ECI$\mathrm{CH}$ ), se procedió a estimar el modelo de regresión múltiple correspondiente (Hair, Anderson, Tatham $\&$ Black, 2004). Se encontró que las cuatro variables eran predictoras significativas de la variable compromiso de la identidad (Tabla 2); el modelo propuesto explica el $14.8 \%$ de la variabilidad.

Como las cuatro variables predictoras en el modelo anterior contribuían a predecir el compromiso de identidad en el Chat, pero no encontramos claros efectos de interacción entre ellas, es que estimamos nuevos modelos de regresión, hasta encontrar efectos de interacción significativos. Se obtuvo que existe interacción entre el tipo de Chat, salud mental (puntaje CSMEJ) y compromiso de identidad en el Chat ( $p=$ 0.004); así los efectos significativos del tipo de Chat $(p=0.02)$ y de la salud mental $(p=0.011)$ sobre el compromiso de identidad en el Chat deben interpretarse a la luz de esta interacción (Tabla 6).

Se estimaron ecuaciones simples que permiten obtener los efectos condicionales de la variable salud mental sobre el compromiso de la identidad, según el tipo de Chat usado. La primera ecuación: $\mathrm{Y}$ $=0.248 . \mathrm{X}+75.053$, donde $\mathrm{X}$ representa al puntaje del CSMEJ, cuando el tipo de Chat es cerrado (error $=0.09, t=2.61, p=0.01)$. La ecuación $\mathrm{Y}=0.857$. $\mathrm{X}+81.934$, siendo $\mathrm{X}$ el puntaje del CSMEJ cuando el tipo de Chat es abierto y/o mixto (error $=0.18$, $t=4.65, p=0.00$ ). Estas ecuaciones indican que la relación entre salud mental y compromiso de la identidad varía según el tipo de Chat (Figura 1). Es decir, en independencia del tipo de Chat usado, los adolescentes pueden obtener puntajes compatibles con la probabilidad de presentar síntomas psico- 
Tabla 6

Regresión múltiple, puntajes de la escala ECICH, puntajes del CSMEJ y tipo de Chat

\begin{tabular}{|c|c|c|c|c|c|}
\hline & \multicolumn{2}{|c|}{ Coeficientes no estandarizados } & \multirow{2}{*}{$\begin{array}{c}\text { Coeficientes } \\
\text { estandarizados } \\
\text { Beta }\end{array}$} & \multirow[t]{2}{*}{$t$} & \multirow[t]{2}{*}{ Significación. } \\
\hline & B & Error típico & & & \\
\hline (Constante) & 75.053 & 1.059 & & 70.860 & $.000 * *$ \\
\hline Salud mental & .248 & .097 & .141 & 2.565 & $.011^{*}$ \\
\hline Tipo de Chat & 6.881 & 2.160 & .156 & 3.186 & $.002 * *$ \\
\hline $\begin{array}{l}\text { Tipo Chat * } \\
\text { Salud mental }\end{array}$ & .609 & .208 & .161 & 2.924 & $.004 * *$ \\
\hline
\end{tabular}

a Variable dependiente: Puntaje total del cuestionario del ECICH

$* * p<0.01$

$* p<0.05$

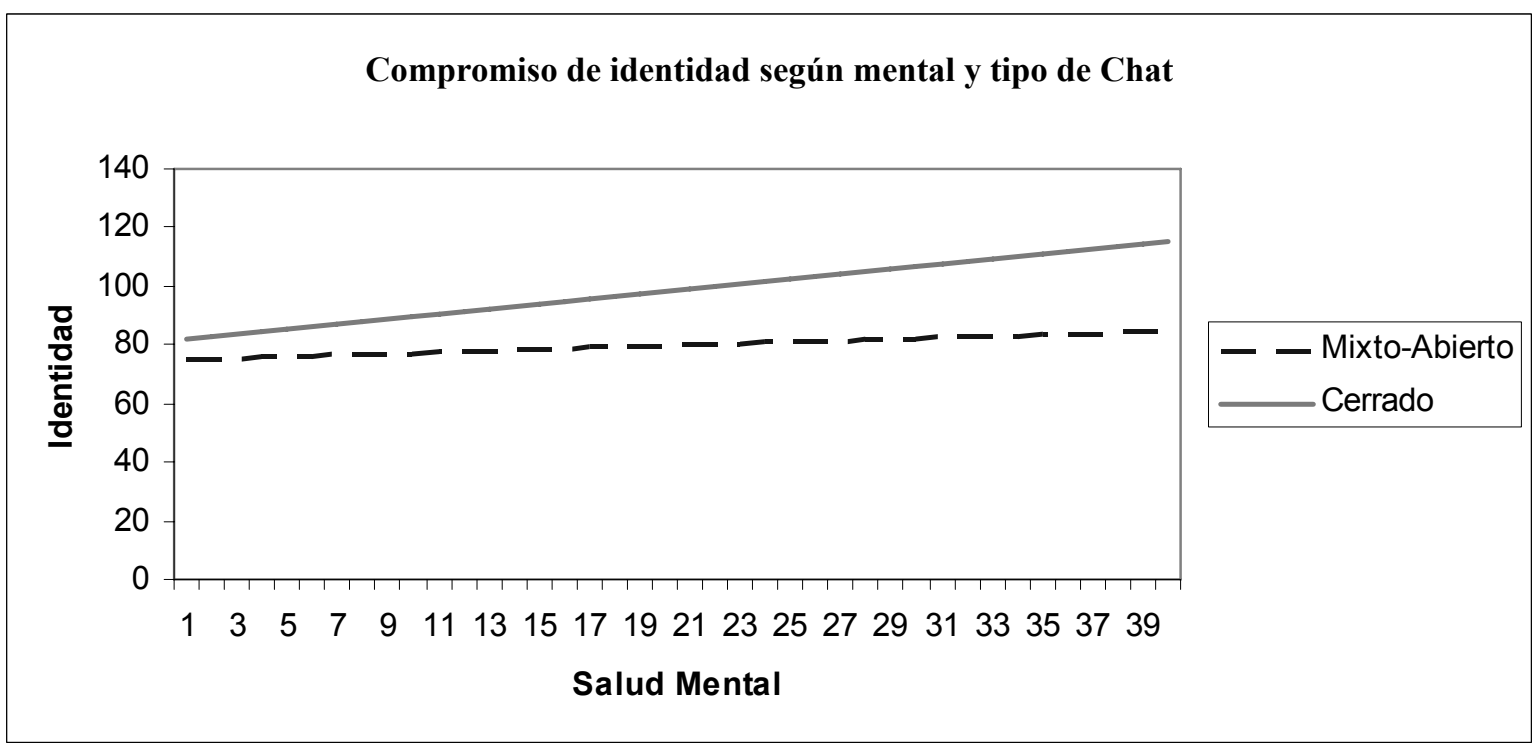

Figura 1. Regresión múltiple, puntajes de la escala ECICH, puntajes del CSMEJ y tipo de Chat.

patólogico; sin embargo, a medida que la citada probabilidad aumenta, se incrementa la probabilidad de que comprometan la identidad en el Chat, incremento que es mayor en el caso de quienes utilizan el Chat abierto y mixto.

\section{Asociación con Probabilidad de Presencia de Sintomas Psicopatológicos, Según Sexo, Curso y Tipo de Chat}

La prueba chi cuadrado de Pearson reveló una relación entre salud mental (puntaje CSMEJ) sólo para la variable sexo, $\chi^{2}(1, N=385)=50.060, p$ $=0.000$. El $25.5 \%$ de las mujeres y el $7.3 \%$ de los hombres del total de la muestra obtuvo puntajes que señalan la probabilidad de existencia de síntomas psicopatológicos. No se encontraron relaciones significativas en relación al curso $\chi^{2}(3, N=385)$ $=0.350, p=0.986$, tipo de Chat $\chi^{2}(1, N=385)=$ $1.341, p=0.247$ y horas de uso del Chat $\chi^{2}(5, \mathrm{~N}=$ $385)=1.550, p=0.907$.

De los que comprometen su identidad en el Chat, un $32.7 \%$ muestra puntajes en el CSMEJ que apuntan a la probabilidad de existencia de psicopatología y en un $67.3 \%$ esta probabilidad no aparecería sugerida. Dentro del $32.7 \%$, un $70.7 \%$ es mujer y un $29.3 \%$ es hombre y se encuentra principalmente 
en I y II Medio; además, un 55.6\% de ellos utiliza predominantemente Chat abierto y un $14.3 \%$, Chat mixto.

\section{Discusión}

La popularidad de los Chat entre los jóvenes nos llevó a preguntarnos si esta comunicación podría relacionarse con el compromiso de la identidad en el Chat.

Si bien, el tamaño de la muestra garantizaba la obtención de una potencia adecuada en los resultados, una limitante se deriva de que estuvo compuesta sólo por alumnos de colegios municipalizados, al variar el grado de digitalización de los menores según el tipo de establecimiento educativo (Fundación Chile, Consultora Adimark \& Empresa de Telecomunicaciones VTR, 2004), aspecto que habrá de tenerse en cuenta en nuevas investigaciones.

Dado el carácter exploratorio de nuestra investigación y porque la literatura consigna diversos problemas metodológicos que la recolección de la información plantea cuando se extrae directamente de Internet (Birnbaum, 2000), decidimos emplear una metodología tradicional. Con todo, futuras investigaciones podrían enriquecerse si usan métodos de análisis cualitativo de mensajes, ya que éstos proporcionan una observación de la interacción entre pares que a menudo "permanece escondida para los adultos en general y para los investigadores en particular" (Greenfield \& Yan, 2006, p. 393).

Optamos por cuestionarios elaborados por investigadores chilenos (CSMEJ y ECICH), porque estimamos constituían procedimientos apropiados para una investigación exploratoria. No obstante, pensamos que nuevos estudios, que escojan una metodología tradicional, podrían profundizar y especificar los hallazgos si emplean otros instrumentos. El CSMEJ no permite identificar patologías específicas, lo que si podría hacerse utilizando un cuestionario como el Diagnostic Interview Schedule for Children, DISC IV (Shaffer, Fisher \& Lucas, 2000). Así se podría aclarar la diversidad de planteamientos respecto al impacto de Internet en el bienestar de los jóvenes y la disminución (Morgan \& Cotten, 2003, Shaw \& Grant, 2002) o aumento de síntomas depresivos (Viñas et al., 2002); así también, respecto de aquellos que presentan ansiedad social (Mc Kenna \& Bargh, 2000; Scealy et al., 2002).

El ECICH no cuenta con puntos de corte específicos que diferencien entre quienes comprometen su identidad en el Chat y aquellos que no lo hacen, tampoco existen estudios de validez concurrente y predictiva. Dado lo anterior, se fijó, provisionalmente, el punto de corte en 86 puntos, como se dijo. Si este puntaje se modificase, los hallazgos de este estudio también podrían cambiar.

Encontramos que el $75.8 \%$ de los jóvenes utiliza sólo Chat cerrado; por sus características, la probabilidad de comprometer la identidad es menor, lo que concuerda con quienes señalan que una de las principales motivaciones para su uso en estas edades es mantener relaciones con amigos, miembros de la familia y de la escuela (Gross, Juvonen \& Gable 2002; Lenhart, Rainie, \& Lewis, 2001 citado en Morgan \& Cotten, 2003).

Tanto el Chat abierto como el mixto son usados preferentemente por alumnos de I y II Medio y como ambos permiten el anonimato, de lo que deriva una mayor posibilidad de experimentación, no es sorprendente si se considera que ellos están en un período de exploración y no han establecido compromisos permanentes; por ende, el medio puede resultarles muy atractivo. Sin embargo, no podemos descartar que alumnos de III y IV Medio, conocedores de los riesgos que la interacción en el Chat abierto trae consigo, la reduzcan o bien oculten información, ajustándose a criterios de deseabilidad social.

El promedio de las horas semanales de uso de Chat fue de 2.19 horas ( $D S$ de 1.63), cifra que indicaría que la muestra estuvo conformada mayoritariamente por jóvenes no dependientes de Internet (Young, 1998). Al igual que Zegers et al. (2004), no encontramos relación entre horas de chateo y compromiso de identidad en el Chat.

No obstante, destaca que un $29.6 \%$ de los jóvenes señaló chatear más de 10 horas semanales, porcentaje superior a los reportados por Zegers et al. (2004), quienes encontraron que sólo el 10.48\% señaló chatear más de 8 horas. Se podría desprender que los patrones de uso cambiarían según edades y nivel de maduración; pero cuando se consideró la distribución de este $29.6 \%$ en los distintos cursos, no se observaron diferencias significativas, por lo que el empleo excesivo del Chat no estaría ligado específicamente a la edad, al menos entre los adolescentes escolares estudiados. Con todo, este $29.6 \%$ puede considerarse un grupo de riesgo respecto de la probabilidad de desarrollar una adicción a Internet. Se infiere, por tanto, la necesidad de que futuros estudios consideren los criterios descritos para el diagnóstico de esta patología (Hall, Alex \& Parsons, 2001; Young, 1998). 
El objetivo general de la investigación fue desarrollar un modelo empírico, descriptivo y predictivo de las relaciones entre compromiso de la identidad en el Chat y posibles variables predictoras en estudiantes de educación media de Santiago de Chile. Se optó por el análisis de la regresión múltiple dado que preliminarmente nuestro interés era predecir la variable criterio: compromiso de identidad en el Chat a partir de las variables salud mental, tipo de Chat, sexo y curso. No obstante, futuras investigaciones podrían usar el modelo de ecuaciones estructurales (SEM) si quisieran considerar distintos niveles de organización de las unidades de análisis (Dilalla, 2000).

Se cumplió el objetivo de desarrollar un modelo empírico, descriptivo y predictivo a través de la regresión múltiple. Tanto la salud mental, el tipo de Chat, el sexo y curso, predicen el grado de compromiso de identidad en el Chat. Además, observamos una interacción entre la variable tipo de Chat usado y la salud mental. De esta manera, si bien los adolescentes que utilizan Chat cerrado podrían comprometer su identidad cuando chatean, la comprometerían menos que aquellos que emplean Chat abierto o mixto. Este hallazgo es un antecedente valioso en el desarrollo de programas que previenen los riesgos que el uso del Chat pueden traer consigo en jóvenes.

En un $32.7 \%$ de la muestra se reunieron los criterios de probabilidad de existencia de síntomas psicopatológicos; de éstos, un $70.7 \%$ eran mujeres, lo cual concuerda con los reportes que establecen mayor prevalencia de sintomatología psiquiátrica entre ellas (Haquin, Larraguibel \& Cabezas, 2004; Vicente, Kohn, Rioseco, Valdivia, Baker \& Torres, 2004). En relación con lo anterior, Almonte y García (2003) sostienen que las adolescentes mujeres están sometidas a mayor estrés, presentan mayor vulnerabilidad a la frustración e inhiben más las manifestaciones conductuales que los jóvenes varones.

Encontramos que el $25.2 \%$ de la muestra compromete su identidad mientras chatea, lo que concuerda con lo planteado por McKenna y Bargh (2000) en el sentido de que la interacción en Internet per-se no produce este compromiso, porque si así fuera, el porcentaje debiera haber sido mayor. Dado que el ECICH evalúa compromiso de identidad en el Chat y que no medimos si estos jóvenes comprometían la identidad en otras áreas de su vida, es que sugerimos emplear algún cuestionario para evaluar solidez de la identidad, por ejemplo, el inventario Identity Consolidated Inventory (ICI) desarrollado por Akthar y Samuel (s/f), usado en este campo por
Larraín, Zegers y Trapp (2007), ya que permitiría distinguir si las experiencias con la identidad en el Chat son una expresión de la exploración propias de la tarea de buscar la identidad (Bargh et al., 2002; Turkle, 1995) o son una consecuencia de un síndrome de difusión de la identidad.

Un cuarto de la muestra compromete su identidad mientras chatea, cifra que es baja en relación al total de jóvenes encuestados; sin embargo, si consideramos los riesgos a los que estos adolescentes se exponen, el porcentaje es alto. Como afirman Zegers et al. (2004), los estados de confusión pueden ser más serios en adolescentes, ya que en esta etapa son más sensitivos respecto a su verdadero sí mismo. Para poder clarificar el alcance de los riesgos antes aludidos se requiere la realización de estudios longitudinales.

De los que comprometen su identidad en el Chat, un $57.7 \%$ obtiene puntajes en el CSMEJ que permitirían descartar la probabilidad de que en ellos existiese alguna psicopatología, mientras que en un $42.3 \%$ esta probabilidad no podría desestimarse. Estos resultados sugieren que efectivamente un porcentaje usa el Chat en el contexto de la exploración de su identidad, mientras que en otros, las motivaciones para su uso podrían vincularse a la existencia de problemas de salud mental. Continuar investigando a este último grupo resulta necesario ya que la presencia de psicopatología en estas edades no siempre es transitoria; puede reflejar reacciones de adaptación menores, pero también puede ser un indicio de síndromes graves y cuyo pronóstico es aún más serio (Noshpitz, 1991; Stein, Fuetsch, Müller, Höfler, Lieb \& Wittchen, 2001).

Respecto de la variable sexo, encontramos que ésta contribuye a predecir la variable compromiso de identidad en el Chat cuando se considera en conjunto con las variables tipo de Chat, edad y salud mental; sin embargo, cuando se trata de establecer la relación de ella sola con compromiso de identidad, no se observa un efecto principal. Tampoco se aprecian diferencias al comparar los promedios entre los sexos en el ECICH. Lo anterior resulta llamativo, ya que la literatura muestra que estas diferencias existen (Herring, 1996; Morahan-Martin, 1998). De allí que estimamos que futuros estudios mantengan el criterio de diferenciación por sexo y de esta manera, establecer si lo hallado en esta investigación se debe a un efecto debido al azar producto del tipo de muestra $o$, efectivamente, las diferencias entre hombres y mujeres están desapareciendo cuando se estudia su comportamiento en el Chat. 
De los datos encontrados, se desprende que el grupo con mayor riesgo de comprometer su identidad en el Chat y que además, muestra mayor probabilidad de que pudiese existir problemas en la salud mental, se encuentra entre las mujeres que cursan I Medio y utilizan el Chat abierto y mixto.

Esperamos con este trabajo haber aportado a un nuevo campo de investigación de la psicología del desarrollo, ya que los psicólogos necesitan comprender cómo los niños y adolescentes participan hoy en día de un universo virtual que es nuevo, masivo y complejo como han señalado Greenfield y Yan (2006).

\section{Referencias}

Acosta, P., Ciunne, L. \& Diodatti, M. (2001). Algunas reflexiones en torno a la adolescencia frente al Chat. Recuperado el 20 de marzo de 2004, desde http://www.fhumyar.unr.edu. ar/ceide/a6.htm.

Akhtar, S. (1992). Broken structures: Severe personality disorders and their treatment. London: Jason Aronson.

Akhtar, S. \& Samuel, S. (1996). The concept of identity developmental. Origins, phenomenology, clinical relevance and measurement. Harvard Review of Psychiatry, 3(5), 254-267.

Akhtar, S. \& Samuel, S. (s/f). Identity Consolidated Inventory (ICI). Manuscrito no publicado.

Allison, B. \& Shultz, J. (2001). Interpersonal identity formation during early adolescence. Adolescence, 143, 509-524.

Almonte, C. \& García, R. (2003). Epidemiología psiquiátrica en niños y adolescentes. En C. Almonte, M. E. Montt, \& F. Correa, (Eds.), Psicopatología infantil y de la adolescencia (pp. 144-170). Santiago: Publicaciones Técnicas Mediterráneo Limitada.

Almonte, C. \& Montt, M. (1999). Descripción clínica y psicopatológica de las principales agrupaciones sindromáticas en psiquiatría infantil y del adolescente. En M. Rizzandini (Ed.) \& C. Saieh (Ed. adjunto), Pediatría (pp. 340-342). Santiago: Publicaciones Técnicas Mediterráneo Limitada.

Altuzarra, M. P. (2006). Relaciones entre uso de Chat, identidady salud mental en adolescentes. Tesis de Magister No publicada, Pontificia Universidad Católica de Chile, Santiago.

Bargh, J. A., McKenna, K. Y. \& Grainne, M. (2002). Can you see the real me? Activation and expression of the "true self" on the Internet. Journal of Social Issues, 58(1), 33-48.

Birnbaum, M. H. (2000). Psychological experiments on the Internet. San Diego, CA: Academic Press.

Castells, M. (1997). La era de la información. Economía, sociedad y cultura. Madrid: Alianza.

Dilalla, L. (2000). Structural equation modeling: Uses and issues. En H. Tinsley \& S. Brown. (Eds.), Handbook of applied multivariate statistics and mathematical modeling (pp. 439463). San Diego, CA: Academic Press.

Douglas, K. \& Mc Garty, C. (2001). Identifiability and selfpresentation: Computer mediated communication and intergroup interaction. British Journal of Social Psychology, 40, 399-416.

Erikson, E. (1959). Identity and the life cycle. Selected papers by Erik H. Erikson. Journal of the American Psychoanalytic Association, 4, 56-121.

Erikson, E. (1968). Identidad, juventud y crisis. Buenos Aires: Paidós.
Estallo, J. (2001). Usos y abusos de Internet [Versión electrónica]. Anuario de Psicología, 32(2), 95-108.

Florenzano, R. (1998). El adolescente y sus conductas de riesgo. Santiago: Ediciones Universidad Católica de Chile.

Fundación Chile, Consultora Adimark, Empresa de Telecomunicaciones VTR. (2004). Los niños cibernautas también son mateos. RTV, 4-7.

Freud, A. (1936/1985). Psicoanálisis del desarrollo del niño y del adolescente (2da. Reimpresión). Buenos Aires: Paidós.

Gergen, K. (1991). El yo saturado. Buenos Aires: Paidós.

Greenfield, P. \& Yan, Z. (2006). Children, adolescents, and the Internet: A new field of inquiry in developmental psychology. Developmental Psychology, 42(3), 391-394.

Gross, E., Juvonen, J. \& Gable, S. (2002). Internet use and well being in adolescence. Journal of Social Issues, 58, 75-90.

Hair, J. F., Anderson, R. L., Taham, W. \& Black, W. C. (2004). Análisis multivariante. Madrid: Pearson Education.

Hall, A., Alex, S. \& Parsons, J. (2001). Internet addiction. College student case study using best practices in cognitive behavior therapy. Journal of Mental Health Counseling, 23, 312-327.

Haquin, C., Larraguibel, M. \& Cabezas, J. (2004). Factores protectores y de riesgo en salud mental en niños y adolescentes de la ciudad de Calama. Revista Chilena de Pediatría, 75, 425-433.

Herring, S. (1996). Posting in a different voice. Gender and ethics in CMC. En Ch. Ess (Ed.), Philosophical perspectives on computer mediated communication (pp. 115-145). Albany: State University of New York Press.

Kroger, J. (2000). Identity development, adolescence through adulthood. California: Sage Publications.

Larraín, M. E., Zegers, B. \& Trapp, A. (2007, 18 de Abril). Do adolescents compromise their identity when chatting? International Journal of Applied Psychoanalytic Studies, 4, Artículo 2. Recuperado el 25 de Junio de http:www3. interscience.wiley.com/cgi-bin/abstract/114211584/ABSTR ACT?CRETRY $=1 \&$ SRETRY $=0$

Lypovetsky, G. (1986). La era del vacio: Ensayos sobre el individualismo contemporáneo. Barcelona: Anagrama.

Maczewski, M. (2002). Exploring identities through the Internet: Youth experiences online. Child \& Youth Care Forum, 31(2), 111- 129.

Mc Adams, D. P. (1993). The stories we live by: Personal myths and the making of the self. New York: The Guilford Press.

Mc Kenna, K. Y. \& Bargh, J. A. (2000). Plan 9 from cyberspace: The implications of the Internet for personality and social psychology. Personality \& Social Psychology Review, 4, 57-75.

Morahan-Martin, M. (1998). Males, females and the Internet. En J. Gackenbach (Ed.), Psychology and the Internet: Intrapersonal, interpersonal and transpersonal implications (pp. 169-197). San Diego: Academic Press.

Morgan, C. \& Cotten, S. (2003). The relationship between Internet activities and depressive symptoms in a sample of college freshmen. Cyber Psychology \& Behavior, 6, 133-142.

Muuss, R. (1996). Theories of adolescence. New York: McGrawHill.

Newman, B. R. \& Newman, P. R. (1999). Development through life: A psychosocial approach (7a Ed.). Belmont, C.A.: Wadsworth.

Noshpitz, J. (1991). Disturbance in early adolescent development. En S. Greenspan \& G. Pollock (Eds.), The Course of life Vol. IV (pp. 119-180). Connecticut: International Universities Press, Inc.

Offer, D. (1991). Adolescent development: A normative perspective. En S. Greenspan \& G. Pollock (Eds.), The 
course of life Vol. IV (pp. 181-200). Connecticut: International Universities Press, Inc.

Reid, E. (1998). The self and the Internet: Variations on the illusion of one self. En J. Gackenbach (Ed.), Psychology and the Internet: Intrapersonal, interpersonal and transpersonal implications (pp. 29-42). San Diego: Academic Press.

Riva, G. (2002). The sociocognitive psychology of computermediated communication: The present and future of technology-based interactions. Cyber Psychology \& Behavior, 5, 581-598.

Sanders, C., Field, T., Diego, M. \& Kaplan, M. (2000). The relationship of Internet use to depression and social isolation among adolescents. Adolescence Magazine, 138(35), 243.

Sandoval, M. (2002). Jóvenes del siglo XXI: Sujetos y actores en una sociedad en cambio. Santiago: Universidad Católica Cardenal Raúl Silva Henríquez.

Sassenberg, K. (2002). Group dynamics: Theory, research, and practice. Group Dynamic, 61, 27-37.

Scealy, M., Phillips, G. \& Stevenson R. (2002). Shyness and anxiety as predictors of patterns of Internet usage. Cyber Psychology \& Behavior, 5(6), 507- 515.

Shaffer, D., Lucas, C. \& Fisher, P. (2000). Computerized diagnostic interview schedule for children (C DISC). New York: Columbia University Development Group.

Shaw, L. \& Grant, L. (2002). In defense of the Internet: The relationship between Internet communication and depression, loneliness, self steem, and perceived social support. Cyber Psychology \& Behavior, 5(2), 157-171.

Stein, M., Fuetsch, M., Müller, N., Höfler, M., Lieb, R. \& Wittchen, H. (2001). Social anxiety disorder and the risk of depression: A prospective community study of adolescents and young adults. Archives of General Psychiatry, 58(3), 251-256.

Subrahmanyam, K., Smahel, D. \& Greenfield, P. (2006). Connecting developmental constructions to the Internet: Identitiy presentation and sexual exploration in online teen chat rooms. Developmental Psychology, 42(3), 395-406.

Suler, J. (1998). Adolescents in cyberspace. Recuperado el 25 de abril de 2004, desde http://www.rider.edu/ suler/psycyber/ adoles.html.

Talamo, A. \& Liborio, B. (2001). Strategies identities in cyberspace. Cyber Psychology \& Behavior, 1(4), 109-122.

Turkle, S. (1995). La vida en pantalla: La construcción de la identidad en la era de Internet. Barcelona: Paidós.

Utz, S. (2000). Social information processing in MUDs: The development of friendships in virtual world. Journal of Online
Behavior, 1(2). Recuperado el 20 de marzo de 2004, desde http://www.behavior.net/JOB/v1n1/utz.html.

Van Hoof, A. \& Raaijmakers, Q. (2002). The spatial integration of adolescent identity: Its relation to age, education, and subjective well-being. Scandinavian Journal of Psychology, 43, 201-212.

Vicente, B., Khon, R., Rioseco, P., Valdivia. S., Baker, C. \& Torres, S. (2004). Population prevalence of psychiatric disorders in Chile: 6-month and 1- month rates. British Journal of Psychiatry, 184, 299-305.

Villani, S. (2001). Impact of media on children and adolescents: A 10 year review of the research. Journal of the American Academy of Child and Adolescent Psychiatry, 40, 392-401.

Viñas, F., Villar, E., Caparrós, B., Juan, J., Pérez, I. \& Cornellá, M. (2002). Internet y psicopatología. El uso del Chat y su relación con diferentes índices de psicopatología. Clínica y Salud, 13, 235-256.

Wallace, P. (1999). The psychology of the Internet. Cambridge: Cambridge University Press.

Wolak, J., Mitchell, K. \& Finkelhor, D. (2002). Close online relationships in a national sample of adolescents. Adolescence, 177(37), 441-456.

Young, K. (1998). Caught in the net. How to recognize sings of Internet addiction and winning strategy for recovery. United States of America: John Wiley \& Sons, Inc.

Young, K. \& Rodgers, R. (1998). The relationship between depression and Internet addiction [Versión electrónica]. Cyber Psychology \& Behavior, 1, 237-244.

Zegers, B. \& Larraín, M. E. (2002). El impacto de la Internet en la definición de la identidad juvenil: Una revisión. Psykhe, 11(1), 203-216.

Zegers, B.; Larraín, M. \& Trapp, A. (2004). El Chat: ¿Medio de expresión o laboratorio de experimentación? Un estudio en una muestra de 124 estudiantes universitarios de Santiago de Chile. Psykhe, 13(1), 53-69.

Zegers, B., Larraín, M. E., Trapp, A., Avilés, M., Grez, V. \& De St. Aubin, S. (2006). Estimación de la validez de constructo y consistencia interna de la Escala de Compromiso de la Identidad en el Chat (ECICH) en una muestra de 123 jóvenes universitarios. Psykhe, 15(1), 79-93.

Zegers, B., Manzi, J., Florenzano, R., Acle, C., Berger, W., González, V. \& Montalbán, J. (1987). Cuestionario de Salud Mental en la Edad Juvenil. Acta Psiquiátrica y Psicológica América Latina, 33, 219-230. 